\title{
Corps et exclusion : la redynamisation du sujet handicapé - chômeur
}

Gilles Bui-Xuân, Anne Marcellini et Jacques Mikulovic

\section{OpenEdition}

1 Journals

\section{Édition électronique}

URL : http://journals.openedition.org/corpsetculture/239

DOI : 10.4000/corpsetculture.239

ISSN : $1777-5337$

Éditeur

Association Corps et Culture

Édition imprimée

Date de publication : 1 juin 1995

ISSN : 1268-5631

Référence électronique

Gilles Bui-Xuân, Anne Marcellini et Jacques Mikulovic, "Corps et exclusion : la redynamisation du sujet handicapé - chômeur », Corps et culture [En ligne], Numéro 1| 1995, mis en ligne le 14 mai 2007, consulté le 08 septembre 2020. URL : http://journals.openedition.org/corpsetculture/239 ; DOI : https://doi.org/10.4000/corpsetculture.239

Ce document a été généré automatiquement le 8 septembre 2020

(c) tous droits réservés 


\title{
Corps et exclusion : la redynamisation du sujet handicapé - chômeur
}

\author{
Gilles Bui-Xuân, Anne Marcellini et Jacques Mikulovic
}

Une expérience instructive

1 Une expérience de suivi et de conduite de stages "d'orientation et d'adaptation à l'emploi" de travailleurs handicapés, chômeurs de longue durée, fait apparaître des résultats appréciables, guère conformes à ceux de stages équivalents. La double originalité du dispositif repose d'une part sur l'introduction dans l'équipe de formateurs d'un éducateur physique, clinicien de l'activité corporelle, et d'autre part sur la supervision d'une équipe d'universitaires (Mikulovic, 1994).

2 La réinsertion professionnelle doit-elle passer par une formation complémentaire, ou bien peut-elle plus particulièrement reposer sur une redynamisation du sujet? Cette question interroge les rapports entre formation et éducation, et suggère d'entrevoir de nouveaux modèles.

3 Cette communication se propose de montrer comment la domination du cognitif dans les systèmes de formation génère de l'exclusion, alors que la gestion éducative d'un modèle conatif pourrait faciliter le retour à l'intégration.

4 Dans cette optique, la question de l'apprentissage dans son rapport au paradigme cognitiviste sera abordée d'abord. Un changement de modèle sera ensuite proposé, différenciant développement - apprentissage - éducation, pour resituer chaque terme dans ce qui pousse le sujet à agir : la conation. Les mobiles différenciés, à l'origine de trajectoires singulières, permettront alors d'identifier des profils-types de stagiaires : consommateurs / transgresseurs / nécessiteux / acteurs.

5 Cette typologie explicite appelle ainsi à reconsidérer les formations.

Le dispositif

6 Dans le cadre du programme européen "Horizon", qui s'intéresse à l'insertion des travailleurs handicapés, des stages" d'orientation et d'adaptation à l'emploi" ont été 
organisés en France et dans divers pays voisins. Dans le bassin d'emplois de Béziers, deux stages consécutifs se sont adressés à des chômeurs de longue durée, reconnus travailleurs handicapés par la COTOREP. Ces stagiaires sont donc entrés dans un nouveau système de formation, devant les conduire à une réinsertion professionnelle.

7 Ces stages ont fait l'objet d'un partenariat entre l'AFPA (Association nationale pour la formation professionnelle des adultes), le CRIP (Centre de rééducation et d'insertion professionnelle), l'AGEFIPH (Fonds pour l'insertion professionnelle des personnes handicapées), et l'Université de Montpellier I à travers l'implication de l'UFR STAPS (Unité de formation et de recherche en sciences et techniques des activités physiques et sportives). Chaque stage a duré 18 semaines, dont 12 semaines de formation et, en alternance, deux fois trois semaines en entreprise.

D'emblée la question des causes profondes des situations très précaires des personnes composant le public concerné est abordée, influençant déjà le projet initial: les supports de formation doivent être prétexte à une "redynamisation des sujets". Certes la plupart des candidats retenus ont eu un "accident" ou une "maladie" du travail; mais si tous les travailleurs bénéficiant d'une indemnité pour $10 \%$ d'invalidité étaient en situation de chômage de longue durée, les statistiques de l'emploi en seraient fortement perturbées. La situation particulière de ces publics laisse donc penser qu'au delà d'un problème de reconversion parfois délicat, notamment dans une conjoncture économique difficile, le choc du handicap a pu provoquer une perturbation psychologique importante. "Un malheur n'arrivant jamais seul", aux problèmes professionnels viennent souvent s'ajouter des problèmes familiaux, matériels, relationnels et affectifs. Ils s'installent alors dans une spirale vicieuse qui les conduit peu à peu à la marginalisation et à l'exclusion.

9 Le but de ces stages est donc moins de donner une formation complémentaire à ces travailleurs (chômeurs) handicapés, que de leur "remettre le pied à l'étrier" et les faire entrer dans une problématique de recherche autonome d'emploi. C'est pour cette raison que, la question de la corporéïté étant abordée, il est fait appel à un intervenant en activités physiques. D'autres tentatives avaient déjà été faites, avec des "moniteurs de sports" ou avec des kinésithérapeutes, sans donner pleinement satisfaction, car installant plutôt les stagiaires dans un rapport instrumental ou rapport d'objet. Le choix de l'intervenant s'est donc porté cette fois sur un étudiant en STAPS de $3^{\circ}$ cycle, présentant la double garantie d'une solide formation clinique d'une part, et d'un suivi méthodologique permanent de son directeur de thèse d'autre part. Un cahier de recherche a donc été ouvert, sur lequel étaient consignées scrupuleusement les observations in vivo et les fragments de verbalisation des stagiaires en cours ou en périphérie de l'activité. S'appuyant sur ce cahier, un compte-rendu d'intervention était alors donné régulièrement, permettant ainsi d'entrer dans un processus de "supervision".

Rapport au cognitif, sentiment d'incompétence et stratégies de masquage

Dans le qualificatif de "travailleur handicapé, chômeur de longue durée", se superposent l'image du travailleur privé d'emploi c'est-à-dire privé du terrain d'expression principal de ses compétences professionnelles, donc privé de son statut et de son identité sociale, et la représentation du handicap qui le range au banc des exclus (Goffman, 1961). Le discours social ambiant renforce sans cesse négativement cette double représentation, si bien que celui qui vit cette situation de l'intérieur ne peut que culpabiliser : Georg Simmel observait déjà en 1892 que "Chaque jour, le nanti chasse le 
mendiant avec colère - comme si c'était moralement un tort que d'être pauvre, comme si cela donc justifiait l'indignation vertueuse. La mauvaise conscience que le riche éprouve en face du pauvre se cache, ici comme tellement souvent, derrière le masque d'une légitimité morale, de façon si continue, avec des pseudo-raisons si péremptoires que la victime elle-même finit par y croire." (p.14). Se sentant donc responsable de sa situation, on peut comprendre que le stagiaire cherche à éviter toute difficulté qui non seulement le stigmatiserait encore plus aux yeux de son entourage, mais qui risquerait également d'augmenter sa propre culpabilité.

11 A cette culpabilité de situation s'ajoute une culpabilité de formation antérieure qui a pu être négligée, et en tout cas le plus souvent mal vécue. Ainsi le retour "à la classe" semble provoquer un sentiment de malaise chez la plupart des stagiaires. D'une façon générale, cela s'exprime par des questions en permanence, mais surtout par des excuses de tout ordre pour pouvoir se dispenser de la tâche exigée : contre-indications médicales, maux divers, interdictions péremptoires, urgences, ou a contrario, manifestations d'hyperactivité. Pour les formateurs, il semble clair qu'à travers ces conduites particulières se joue la préservation d'une identité ancienne et acceptée (Demazière,1992).

12 L'originalité de l'intervention de l'éducateur physique repose sur une absence totale d'exigence de performance ou d'expression de savoir-faire établis. Bien sûr la première manifestation des stagiaires est un étonnement général de devoir pratiquer "du sport" alors que justement leur handicap le leur proscrit. Les dispenses d'activité physique pour raison médicale sont d'entrée brandies. Qu'à cela ne tienne, le profil des stagiaires est bien connu et c'est justement la raison pour laquelle est organisée cette animation corporelle! Les dispenses sont donc refusées en bloc, et l'explication de l'éducateur, si elle laisse sceptique, permet toutefois la mise en branle du groupe.

Car c'est bien d'une activité de groupe dont il s'agit, chacun se réfugiant derrière l'ensemble pour ne pas être mis en demeure de démontrer ce qu'il sait faire. Il conviendrait plutôt de dire "ce qu'il ne sait pas faire", car c'est bien là la question qui semble hanter chacun d'entre eux. La représentation qu'ils ont du "sport" semble basculer d'une vision scolaire à l'image du haut niveau, mais elle reste toujours empreinte de la nécessité d'un apprentissage dont il faudrait rendre compte. Et, disentils: "ils ne savent pas, car ils n'ont pas appris!" Bien que jamais la consigne de l'éducateur physique n'ait exigé cela, cherchant au contraire à ce que s'expriment spontanément les potentialités de tous ou les schémas intégrés de chacun, il faudra du temps avant que sa demande soit entendue.

En réalité, ce que manifestent inconsciemment les stagiaires, c'est l'expression d'un vaste sentiment d'incompétence dont ils sont épris, et dans lequel, en activité physique comme ailleurs, ils s'enferment peu à peu. Il faut dire que le cumul des échecs successifs ne peut que favoriser le développement de ce sentiment.

Le développement des modélisations systémiques, à partir de l'émergence de la cybernétique, amène aujourd'hui à penser différemment les processus d'émergence et de renforcement du handicap. L'intérêt porté dans cette logique aux interactions et en particulier à l'idée de réciprocité (feed-back) a donné lieu à la modélisation de ce que l'on peut nommer "cercle vicieux", ou "effet en spirale". Dans un système d'interactions, la prédominance et la récurrence de rétroactions positives (toujours plus du même) amène à "l'emballement" du système qui n'est plus "régulé" (Watzlawick, 1972). D'un point de vue strictement physiologique, la modélisation de ce 
que les québécois, en particulier, appellent le syndrome H/H (Hypokinésie/ Hypodynamie qui aboutit au déconditionnement physique) peut se décliner à l'infini sur différents plans, offrant ainsi des visualisations on ne peut plus parlantes de la réalité en général. Ainsi dans le champ très spécifique du handicap et des inadaptations le repérage de tels "mécanismes en spirale" peut se faire sur les plans : physique $(\mathrm{H} / \mathrm{H})$, cognitif (échec répétitif/sentiment d'incompétence qui s'aggrave et qui génère de l'échec), social ou psychosocial (dépendance/prise en charge du sujet qui aggrave sa dépendance; identité négative/sentiment d'inutilité qui renforce la connotation négative de l'identité), et affectif (souffrance affective/désorganisation affective qui contribue à la souffrance affective). Cette logique d'installation dans le handicap est en outre complexifiée par le fait que ces différents plans d'analyse sont évidemment euxmêmes en interaction réciproque dans le système que constitue chaque sujet humain.

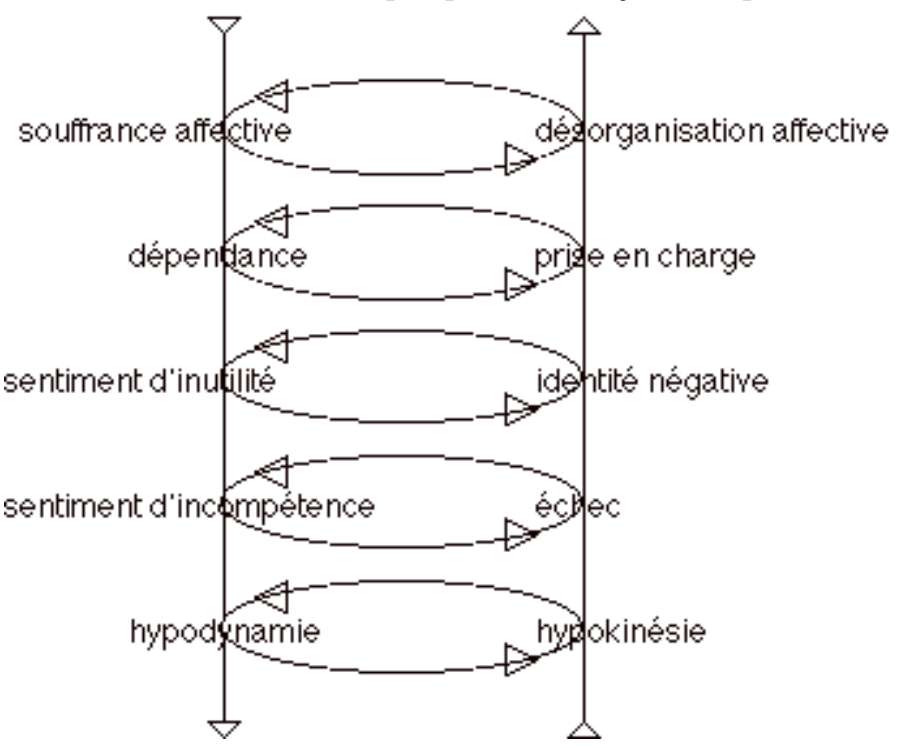

Schéma 1 : différents plans interactifs

La spirale "sentiment d'incompétence/échec" est ici particulièrement éclairante : pour faire face au discrédit, c'est-à-dire à la révélation de leur incompétence aux yeux des autres, les stagiaires déploient tout un arsenal de routines habituellement bien rodées pour déjouer l'attention de tout observateur. Cependant cette remarque n'aurait pu être clairement formulée si, justement en activité physique, ces stratégies de masquage avaient pu comme ailleurs être mises en oeuvre. Mais cette activité étant d'une part parfaitement inhabituelle, et sa conduite étant d'autre part tout à fait orientée sur la réussite des stagiaires et non leur mise en difficulté, aucune réponse toute faite, ni de détournement de la tâche, ni de démonstration intempestive de compétences factices, ne pouvait être déployée.

Devant cette impuissance à faire face dans les formes accoutumée la parole était la dernière bouée à laquelle ils se raccrochèrent pour préserver une identité acceptable. Sentiment d'incompétence et stratégies de masquage furent donc révélés à l'ensemble de l'équipe de formateurs, qui crièrent qu'à l'évidence il en était de même dans leurs disciplines respectives. Encore suffisait-il de le dire pour s'en rendre compte. Le problème demeurant que dans les disciplines traditionnelles les stratégies de masquage sont suffisamment bien rodées pour passer inaperçue.

La prégnance du cognitif dans la qualification 

représentait pas une vaste situation handicapante, on peut penser que pour ces publics en difficulté d'intégration sociale comme scolaire, les situations traditionnelles de formation les placent inexorablement en situation de handicap.

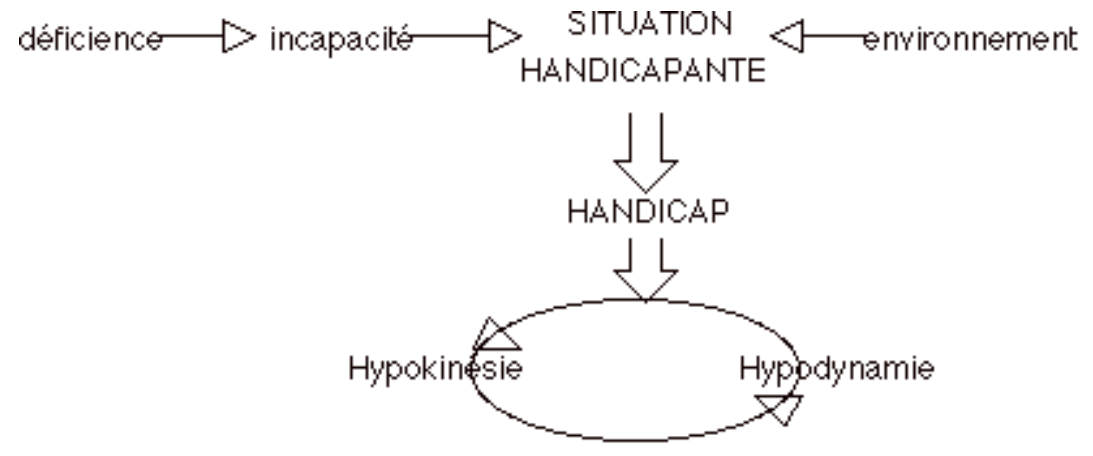

Schéma 2 : L'enfermement dans le handicap pouvoir envisager, d'un point de vue théorique tout d'abord, les possibilités de remédiation ou tout au moins de régulation permettant de sortir de "la spirale" du "toujours plus de handicap". En effet, la théorie interactionniste met en évidence que dans un système, tout changement dans le réseau des interactions provoque inévitablement d'autres changements en cascade dans l'ensemble du système. Il suffit alors, pour sortir des situations "bloquées" sous la forme de cercles vicieux de générer un changement dans une interaction donnée pour susciter une réorganisation plus large de l'ensemble du système. Dans ces conditions, et dans les conditions "exceptionnelles" d'un stage, pour réduire le handicap, il s'agirait d'abord de penser la transformation de l'interaction sujet/environnement. Qu'est-ce qui fait que cette interaction est réellement handicapante pour certains sujets? Et comment la modifier pour qu'elle ne le soit plus?

21 Si l'apprentissage peut représenter un passage obligé dans le développement d'un curriculum menant à l'expertise, le "bonheur d'apprendre" ne peut concerner qu'une population qui a dépassé l'étape des apprentissages purs pour se mouvoir dans un contexte plus complexe ou se projeter dans un avenir prometteur (Bui-Xuân, 1993). En dehors de l'adhésion à une conformité de bon aloi, pour ceux-ci l'obligation d'apprendre a dû tomber, bon gré mal gré, à une étape légitime de la maturation de leurs significations. Mais pour les autres, pour tous ceux pour qui l'apprentissage pèse, non pas qu'ils aient du mal à apprendre, mais plutôt du mal à comprendre et à accepter qu'il faille apprendre, le devoir d'apprendre ne saurait être vécu que comme un malheur.

Or, si le fonctionnement humain permet de percevoir des indicateurs synthétiques de situation, des figures du social (Tacussel, 1993), alors il importe de déceler les indices perceptibles qui révéleraient qu'une situation de formation est véritablement handicapante pour certains. Les rapports à l'apprentissage, à l'acquisition de 
connaissances comme à l'intégration de savoir-faire ou de techniques, mais connaissances ou techniques pour elles-mêmes ou pour leur simple restitution, pourraient représenter un bon révélateur de discrimination. Dans un environnement coercitif où domine le pouvoir de la connaissance, il y a ceux qui veulent se l'approprier et ceux qui ne lui accordent ni sens ni intérêt immédiat et pour qui elle représente un véritable obstacle de vie.

Ce sont ceux-ci qui illustrent le mieux la transposition du "syndrome $\mathrm{H} / \mathrm{H}$ " du plan physiologique au plan cognitif : la perpétuation d'un rapport négatif à l'apprentissage fait entrer la personne dans la spirale du déconditionnement cognitif, "moins j'apprends moins j'ai envie d'apprendre".

24 Ainsi tout cadre de formation, quel qu'il soit, devrait s'interroger sur le rapport entre ses exigences en matière d'apprentissage, et le sens que ces apprentissages revêtent véritablement chez les personnes en formation. Il devrait également se questionner sur le décalage éventuel entre ce sens et celui conféré par les formateurs eux-mêmes au regard de la discipline enseignée. Ainsi, si la prégnance du cognitif génère de l'exclusion, ce nouveau regard pourrait être à l'origine d'un changement de paradigme en matière de formation.

A.P.S, conation et restauration d'un sentiment de compétence

On a vu que, confrontées à un même environnement, certaines personnes le perçoivent comme un obstacle rédhibitoire alors que d'autres solutionnent les problèmes qu'ils $\mathrm{y}$ rencontrent. Si pour les premiers le handicap naît bien de cette interaction d'où émergent des situations qui sont handicapantes pour eux seuls, c'est l'exemple des seconds qui conduira l'intervention pédagogique : s'ils n'intègrent pas le cercle $\mathrm{H} / \mathrm{H}$, leur mode de fonctionnement devrait éclairer ceux qui veulent en sortir. Mais qu'est-ce qui les différencie fondamentalement, si ce n'est qu'ils n'entretiennent pas le même rapport à cet environnement ?

26 Le principe utilisé pour rompre le cercle vicieux du syndrome $\mathrm{H} / \mathrm{H}$ repose donc sur la transformation ou l'aménagement d'un environnement qui, au lieu d'installer ou de suggérer un obstacle rédhibitoire, positiverait la personne en la plaçant de fait en situation de réussite, de mobilisation, et par là de responsabilité. Il est évidemment possible d'engager ce principe à partir de n'importe quelle dimension de la personne, comptant sur l'interaction des différentes composantes pour espérer un effet sur l'ensemble. Mais c'est bien en activité physique que le coup d'envoi est donné, car ailleurs des résistances sont élaborées par chacun. Et comme il n'existe guère d'objets pédagogiques adaptés à l'ensemble d'une population, chacun peut se réfugier derrière des paravents: qu'il s'agisse de tactiques de masquage ou de démonstrations intempestives, l'objectif est toujours le même; il faut éviter l'évaluation, c'est-à-dire le regard objectif de l'autre.

On peut comprendre qu'une personne investie d'un vaste sentiment d'incompétence puisse anticiper sur un échec prévisible et se soustraire à toute situation qui pourrait la mettre en demeure de réaliser une tâche évaluée. Alors l'application d'un programme de mobilisation, réussie, progressive et évaluable peut contribuer à la restauration d'un sentiment de compétence qui risque fort de redynamiser le sujet en vue de nouvelles entreprises.

28 La place du regard est donc primordiale dans cette intervention, comme dimension déterminante de l'environnement. Le simple fait d'énoncer, de dire ce que fait quelqu'un, en le positionnant dans une dynamique évolutive comme un moment 
particulier, relevant à la fois de sa singularité et de sa responsabilité, c'est déjà le reconnaître comme sujet, acteur de sa propre construction. Situer une personne dans un curriculum conatif participe de ce projet (Bui-Xuân, 1994).

Mais deux conditions principalement font que l'application de ce modèle en APA (activité physique adaptée) convient particulièrement bien. La première est que toutes les classifications traditionnelles décrivent la Personne handicapée comme une personne ordinaire à qui il manquerait quelque chose. Or n'importe qui se structure spécifiquement en fonction de ce dont il dispose, et non pas par rapport à ce qu'il n'a pas (Bui-Xuân, 1987). D'ailleurs comment pourrait-on objectivement évaluer, voire tout simplement envisager quelque chose qui n'existe pas? Or le modèle conatif ne spécule pas sur le manque, mais décrit ce qui est fait en en cherchant le sens. La deuxième est que toutes les listes d'activités ou "d'habitudes de vie" ne sont pas en mesure de rendre compte du rapport spécifique que peut entretenir un sujet quel qu'il soit avec chacune d'entre elles. A l'heure actuelle, seule une classification des jeux et des sports est en mesure de réaliser cette correspondance.

De plus, tout le monde a un corps et sait en apprécier autant le potentiel que la singularité. Et cependant, bien qu'il soit parfaitement en mesure de se situer par rapport aux autres, il n'est jamais mis en demeure d'en faire la démonstration. Cette inexpérience de la confrontation est la raison pour laquelle il n'a pas, dans ce domaine, élaboré de stratégie ni de tactique de masquage et de détournement de la prestation demandée. A partir de l'activité corporelle donc, l'objectif est moins de recomposer une nouvelle classification des handicaps ou des personnes handicapées, que de confronter ces sujets à une classification de dispositions pour déboucher sur une véritable "évaluation" positive en observant ce qu'ils réalisent en situation réelle de pratique (Bui-Xuân, 1993). L'intérêt majeur de cette évaluation est qu'elle ne saurait être ni figée ni définitive, dans la mesure où elle se présente comme un moment dans un curriculum, porteur de projets et de progrès. Elle peut jouer le rôle d'une évaluation diagnostique, aussi bien que formative et formatrice, mais surtout pas sommative.

En effet, construite dans la perspective du suivi de l'évolution singulière d'un sujet dans son rapport avec l'activité, elle ne se réfère à aucun barème et tente d'engager le sujet lui-même comme acteur du processus évaluatif.

L'énorme avantage que présentent les activités physiques et les sports, c'est que leur diversité est telle qu'il serait étonnant de ne pas trouver une activité ou une modalité d'activité qui ne corresponde pas à la combinaison spécifique des qualités d'un sujet. De surcroît, les modalités de la victoire ou de l'excellence permettent d'identifier clairement le sens et l'essence de l'activité, son principe directeur et les qualités dominantes qu'il exige: mesure et structure physiques, conformité à un modèle et savoir-faire techniques, ou score et fonctionnalité mentale et/ou motrice. Le sens historique des jeux et des sports s'ancre en effet dans un imaginaire collectif qui distingue bien dans la performance, ce qui est de l'ordre du développement du sujet, de ses apprentissages ou de son éducation. 


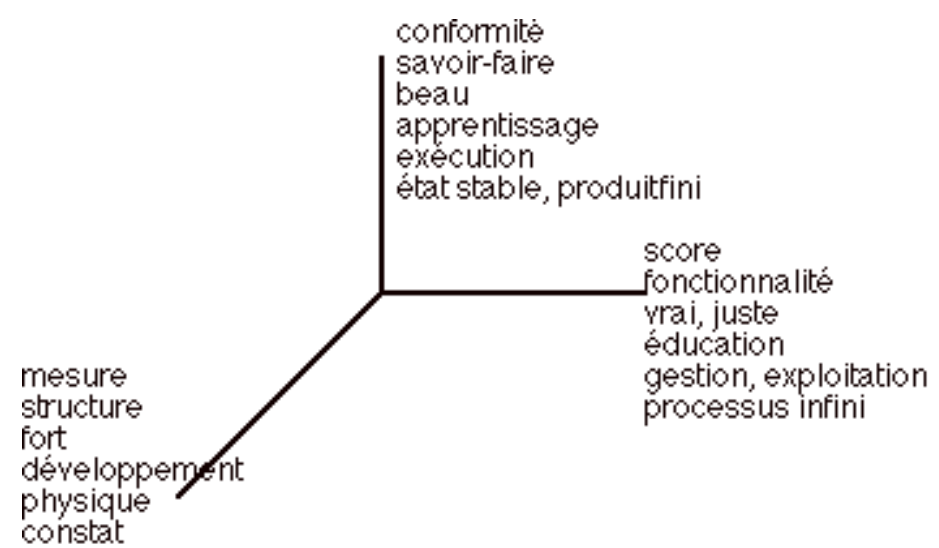

Schéma 3 : définition des axes de la modélisation (Bui-Xuân 1994)

Après avoir établi des corrélations très significatives entre la fonctionnalité des sujets, repérée par des conduites-types au regard d'un curriculum conatif en situation réelle d'activité, et d'une part leurs capacités physiques (structure), et d'autre part leurs savoir-faire dans l'activité (techniques), il est alors possible de proposer une mise en correspondance du "volume" de personnes et du "volume" d'activités sportives, de façon à ce que chacun puisse pratiquer une activité physique adaptée, à sa mesure.

Une fois l'activité choisie, le curriculum conatif commun permet d'éclairer un référentiel particulier, et de situer le pratiquant à l'une ou l'autre de ses étapes (émotionnelle, fonctionnelle, technique, de contextualisation et d'expertise). En révélant à l'observateur la préoccupation majeure du sujet, c'est-à-dire l'état du rapport signifiant ponctuel qu'il entretient avec le principe directeur de l'activité, cette nouvelle grille dynamise l'action classificatoire, en relativisant la place du sujet qui "évolue" dans son rapport à l'activité en question. Elle propose ainsi un changement de paradigme en positivant l'action du sujet.

5 Ce nouveau regard sur le sujet, en positivant sa place dans le curriculum, autorise un nouveau regard du sujet sur lui-même. En acceptant sa projection dans un futur favorable, il facilite la maturation de ses significations. Et si ce sens évolue, alors le sujet élaborera de nouvelles règles de fonctionnement, et par là même adoptera de nouveaux modes de vie. Ainsi, la résultante de la confrontation du sujet à son environnement a changé, et par là l'ensemble du système.

Si les classifications usuelles limitent l'action de l'intervenant, sans qu'il ne puisse rompre le cycle "hypodynamie-hypokinésie" (Simard et al., 1993), c'est parce qu'elles renvoient au "manque" à combler. En considérant d'entrée que toute personne, qu'elle soit handicapée ou non, peut pratiquer un sport à sa mesure, il s'agit alors de mettre en correspondance les capacités évaluées du sujet et les exigences des activités sportives. Le choix judicieux d'une APS permet au sujet de mobiliser ses ressources et de s'engager dans l'activité en question, rompant ainsi son attitude hypokinétique. L'évaluation que font le sujet et son entourage de sa prestation, en le situant dans une étape conative, relativise sa conduite sportive au regard de toute norme, et positive son action effective. Le sujet est ainsi redynamisé par cette mise en évidence de son pouvoir d'agir, rompant ainsi avec son attitude habituelle d'hypodynamie.

Le syndrome $\mathrm{H} / \mathrm{H}$ est alors rompu, et le passage d'un cercle vicieux à une spirale vertueuse peut alors être envisagé. 
Les résultats, tant quantitatifs que qualitatifs, corroborent magistralement l'hypothèse selon laquelle l'application de ce modèle favoriserait la réinsertion professionnelle de cette population d'exclus du système de production. Il convient toutefois de nuancer les plus de $80 \%$ d'embauche d'une part en relativisant la nature de celle-ci (CDI, CDD, CES...), et d'autre part en veillant à s'assurer de sa pérennité dans le temps.

Compétences diverses et stratégies individuelles

L'analyse psychosociale des situations de dévalorisation en général (recherches sur les immigrés, les chômeurs, les populations très défavorisées) (Malewska-Peyre, 1990), et des situations de handicap en particulier met en évidence différents types de réactions à l'assignation à une position sociale dévalorisante. On repère dans ces situations sociales spécifiques des positionnements identitaires dits "positifs" et d'autres dits "négatifs", les premiers renvoyant à une remise en cause de l'identité sociale assignée, les seconds à une acceptation de l'identité sociale assignée, intégrant la connotation négative (Demazière, op.cit.). Le maintien, ou les tentatives de maintien d'une identité positive s'actualisent au travers de la mise en place de stratégies identitaires qui visent à un changement de la réalité qui s'avère stigmatisante (position active). L'acceptation d'une identité négative renvoie à une installation dans des stratégies de confirmation de la réalité stigmatisante (position passive) (Marcellini, 1991).

L'analyse des différents types de réactions à une désignation sociale stigmatisante montre que l'adoption d'une situation active ou passive face à une telle agression renvoie à l'adoption soit d'une position de sujet-acteur du système social, soit de celle d'un objet-utilisateur du système social. Dans la situation particulière de dévalorisation identitaire que constitue l'assignation au handicap, la position de sujet-acteur se joue au travers du refus de l'intégration aux groupes des handicapés (refus de l'attribut handicapé), ou par l'engagement dans un militantisme visant au changement des représentations sociales du handicap (refus de la connotation négative associée au handicap) (Marcellini, op. cit.). La position d'objet-utilisateur se concrétise par un usage social du handicap et des structures d'assistance sociale qui y sont associées. C'est dans le cadre d'une étude psychologique approfondie de chaque cas, complétant l'approche psychosociale, que des interprétations de l'adoption de l'une ou l'autre des positions par un individu pourraient être avancées. Mais dans le cadre d'une réflexion appliquée, utilisable dans le champ de l'action sociale, les rôles de sujet-acteur et d'objet-utilisateur permettent de penser les principes directeurs d'une intervention pédagogique qui viserait à la sortie de la "spirale" négative. 


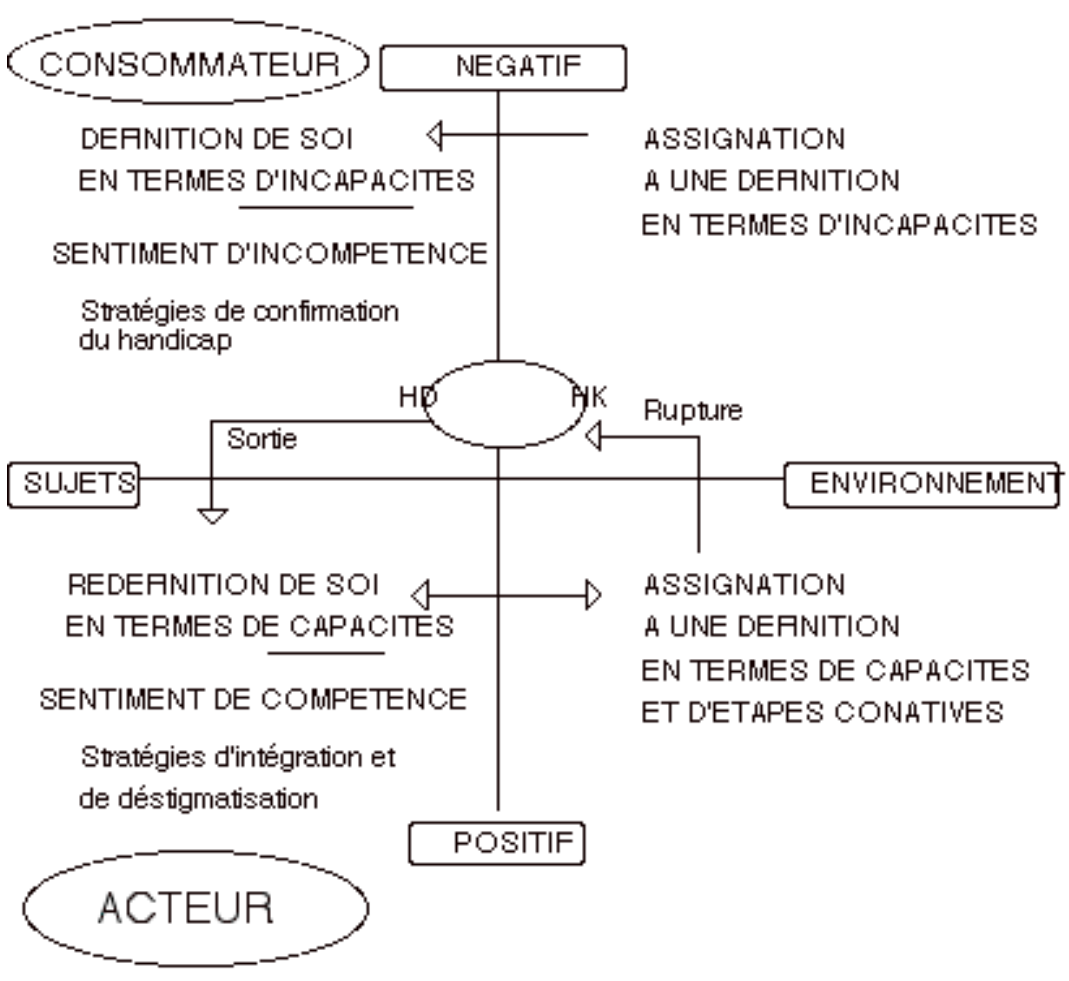

Schéma 4 : positiver pour une redéfinition du sujet

- le handicapé consommateur : il est en retrait et renonce à toute revendication, si ce n'est celle de recevoir une allocation qu'il considère comme un dû. Il se conforte dans son statut de handicapé, et reste passif et inactif dans la recherche d'un emploi.

- le chômeur type : tous les malheurs du monde lui sont tombés dessus, et l'accident ou la maladie l'ont achevé. Si ses initiatives ne sont guère pertinentes, il reste cependant disponible et intéressé. L'assistance peut être transitoire, car avant tout, il a besoin de prendre confiance.

- le transgresseur, ou bénéficiaire du système : il parle beaucoup de ses malheurs et de ses compétences. Mais son hyperactivité masque son désengagement. En fait il est débrouillard et a des sources de revenus qu'il ne dévoile pas.

- le handicapé "volontaire" : le handicap l'a détourné de sa profession originelle, mais il reste dynamique et véritable demandeur d'emploi, disposé à se réinsérer professionnellement. Pour lui le stage devrait être un tremplin.

Cette catégorisation devrait permettre une meilleure orientation des sujets. En effet, la redynamisation s'est avérée efficace avec seulement deux types de personnes: les handicapés "volontaires", et les "chômeurs types". Les deux autres classes se sont montrés réfractaires à la plupart des propositions pédagogiques. Les "handicapés consommateurs" et les "transgresseurs" ne sont en effet guère à leur place dans ces stages. Les premiers ne sont pas en crise identitaire, et l'assignation au handicap non seulement ne les gêne pas, mais ce serait plutôt le contraire qui les contrarierait. Quant 
aux seconds, il est probable qu'ils ne soient pas imprégnés d'un sentiment d'incompétence, ni pris dans la spirale de l'échec. S'ils déploient des stratégies de masquage, il s'agit du masquage de leurs réelles compétences : compétences inavouées officiellement parce qu'illicites d'une part, et compétences inavouables parce qu'au banc des pratiques sociétales d'autre part.

Ainsi, parmi ces personnes qui sont reconnues administrativement "travailleurs handicapés, chômeurs de longue durée", il y aurait les vrais, ceux qui sont chômeurs parce que le handicap les a touchés et les place en position d'exclus; et les faux, ceux qui revendiquent leur handicap pour pouvoir continuer à profiter du chômage : mais ces derniers sont-ils vraiment des exclus?

Il est possible de sortir du système professionnel officiel, et jouir d'une intégration sociale tout à fait satisfaisante. Nirje (1980) distingue cependant un certain nombre de niveaux d'intégration : l'intégration physique, l'intégration fonctionnelle, l'intégration sociale, l'intégration personnelle, l'intégration sociétale, et l'intégration organisationnelle. Bien sûr, si le travail fonde tout son système de relation, alors sa rupture risque fort d'entraîner une rupture d'intégration sociale. Mais si ce n'est pas le cas, et qu'une personne ne travaille pas ou plus sans pour autant perdre de vue ses relations sociales, alors c'est au plan de l'intégration sociétale que tout se joue. Soit cette personne estime devoir jouer un rôle dans la cité, être un des acteurs de la vie de la société, et sa prise en charge en cas de difficulté ne peut être considérée que comme passagère, provisoire, tremplin pour retrouver une place à travers laquelle sa participation à la construction de l'ensemble du système puisse être assumée; soit la notion de devoir lui échappe et elle considère plutôt que la société lui est redevable de quelque chose, et qu'en conséquence elle doit payer. Le choix d'une personne dans cette alternative ne saurait dépendre de ses compétences personnelles ou professionnelles: c'est une question d'éducation des fonctions sociétales. L'étude sur ces populations n'est pas encore en mesure de faire ressortir s'il s'agit d'une éducation de base, dont la trace pourrait être suivie tout au long de la trajectoire de vie d'une personne, ou bien si un événement exceptionnel peut rompre et détourner ces fonctions. En attendant, il est clair que l'exclusion n'est pas vécue de la même façon par quelqu'un qui pense devoir tenir une place comme rouage du système, et un autre qui n'a jamais cherché à prendre rang (Wuhl, 1992).

Dans ces conditions, qu'est-ce que des stages de la sorte peuvent-ils apporter?

Le rapport aux fonctions sociétales pourrait représenter un bon indicateur d'utilité.

En effet, avec les sujets caractérisés de "handicapés qui en veulent" ou de "chômeurstypes", la rupture sociétale n'est pas consommée et le stage peut jouer son rôle de dynamiseur, de catalyseur, et redonner confiance tout en les remettant en situation d'activité professionnelle réussie (Sainsaulieu, 1988). Pour ces personnes qui ont accepté de "jouer le jeu" et qui sont entrées de plain pied dans des propositions pédagogiques qui leur ont permis de se voir positivement tout en faisant le point sur leurs compétences, les résultats sont remarquables.

Mais ce sont les autres, ceux qui ont été caractérisés de "handicapés consommateurs" ou de "transgresseurs", qui ont mis en évidence les limites du système : refusant le plus souvent "d'entrer dans la danse" au sens propre comme au figuré, ils ont rapidement manifesté un mépris pour ce type de formation qui leur paraissait illusoire. Forts de compétences déclarées, mais refusant d'être évalués et de se voir objectivement, ils ont 
mis en demeure les formateurs de leur trouver stages et emplois, sans pour autant fournir d'efforts pour les rechercher eux-mêmes. Toujours mécontents des propositions des formateurs, ils agissaient comme si un emploi idéal leur était dû. Pour ceux-ci les résultats se sont avérés assez maigres.

Ainsi le rapport aux devoirs sociétaux semble bien déterminant pour l'accès à un emploi après ce type de stage de réinsertion : il semble assez difficile de faire sortir les "consommateurs" et "transgresseurs" de leurs relations objectales et de leurs manipulations des formateurs et du système; en revanche "nécessiteux" et "acteurs", en accédant ou en maintenant une relation de sujet, peuvent établir un rapport suffisamment positif aux intervenants et au système pour trouver une nouvelle place dans la production et dans la société.

Formateur ou éducateur?

51 Ces remarques ne peuvent qu'interpeller les décideurs institutionnels sur le bien-fondé et l'usage qui est fait des stages de formation ou de qualification destinés aux chômeurs. S'il s'avérait qu'une réelle formation soit nécessaire pour accéder à un emploi spécifié, encore faudrait-il se demander si les difficultés de tous ordres face aux apprentissages ne représentent pas un obstacle rédhibitoire pour des personnes justement en situation d'échec. Quant à celles qui affichent des compétences répertoriées, sont-elles en mesure de quitter ces structures de formation une fois la qualification acquise?

Les résultats assez médiocres des stages de qualification, du moins sur le plan de l'embauche, laissent penser qu'ils peuvent recouvrir une autre fonction : rendre, aux yeux de ceux qui travaillent, les chômeurs responsables de leur inactivité. Il sera alors possible de distinguer ceux qui ne peuvent pas accéder à la qualification qu'on leur offre pourtant, et qui ainsi ne peuvent prétendre à l'emploi, et ceux qui, jugeant qu'ils ne sont pas encore assez qualifiés pour prétendre à l'emploi, diffèrent leurs recherches d'embauche à un avenir meilleur tout en accumulant les stages de formation.

Ces considérations pourraient laisser entendre que la formation des chômeurs ne serait qu'un prétexte socio-politique.

On a pu voir cependant que sous couvert d'un stage de formation, c'était l'occasion d'une véritable éducation qui pouvait être envisagée : elle ne s'adresserait qu'aux uns, à ceux qui, certes en détresse affective et/ou cognitive, seraient suffisamment investis de sentiments sociétaux pour entrer dans le jeu d'intervenants qui se proposeraient de mobiliser et d'optimiser les fonctions motrices et mentales de gestion et d'exploitation de leurs capacités propres.

Pour les autres cependant, c'est-à-dire les consommateurs de stages, ceux qu'aucun sentiment sociétal n'anime, la "formation" pourrait représenter le dernier rempart d'une éducation, ou peut-être d'une instruction, éthique. Mais en la matière, en dehors des camps de rééducation, les expériences manquent (!).

Face à cette impossible formation, les "formateurs" ne sont pas dupes. Habitués aux procédures d'apprentissage, qu'ils ont eux-mêmes intégrées tout au long de leur trajectoire qui les a mené de l'entreprise au centre de formation professionnel, ils s'aperçoivent vite qu'aucune de ces procédures ne saurait être efficiente avec ces populations en grande difficulté. De façon empirique ils entrent alors dans une relation pédagogique plus clinique, interprétative et éducative que comportementaliste, technique et instructive. Il faut dire que l'intervention et la rencontre avec des 
universitaires ne les a pas détournés de cette intuition première et les modèles développés les ont confortés dans ce changement d'orientation.

S'inscrivant déjà dans une dynamique "distinctive" de positionnement social, il semble que celle-ci soit renforcée par leur adhésion à cette nouvelle mission éducative (BuiXuân, 1987). Il reste à savoir si cette identité d'éducateur survivra aux stages particuliers "d'orientation et d'adaptation à l'emploi" de travailleurs handicapés, chômeurs de longue durée.

Conclusion : le handicap comme traitement social du chômage

Dans le seul bassin d'emploi de Béziers, 940 travailleurs handicapés sont inscrits à l'ANPE. Ces chiffres ne peuvent que croître au fur et à mesure que se développe le chômage. Face à une telle situation, les pouvoirs publics seront amenés à déployer tout un arsenal de mesures, visant non seulement un traitement social du chômage, mais aussi une adaptation aux caractéristiques particulières des chômeurs. Ces stages initient une série de décisions qui risquent fort de se révéler à terme peu efficientes si elles ne prennent pas en considération les bilans des premières expériences.

Parmi ces conclusions, il doit être noté la force des représentations dominantes en matière de formation, et particulièrement le primat de la connaissance, théorique et/ ou pratique.

60 Paradoxalement, le modèle conatif a permis de redynamiser et par là de réinsérer professionnellement ceux qui, pris dans la spirale vicieuse de l'échec, étaient les plus en difficulté cognitive. Pourtant conduits à optimiser leurs capacités propres, ceux-ci ne surent pas abandonner une demande explicite d'accumulation de connaissances qui s'avéraient parfaitement inutiles pour leur nouveau métier. Dans la perspective de ce nouveau départ, le handicap était relégué au second plan.

61 En revanche, ceux qui sont les plus convaincus d'être compétents, et qui de fait sont bien les plus calculateurs et les plus stratèges, ne s'estiment jamais suffisamment qualifiés pour quitter le système de formation, et s'installent dans le chômage. Le handicap est alors mis en avant comme tactique d'évitement des offres d'emplois.

62 Mais vaut-il mieux accorder une allocation de "travailleur handicapé" ou faire grimper les statistiques du chômage?

63 Les "vrais" handicapés, refusant cette logique, dissimulent les stigmates du handicap pour réintégrer le monde économique.

64 Quant aux "faux", ils préfèrent adhérer à la figure sociale du handicap pour mieux justifier leur exclusion économique.

65 Un effet pervers du système d'assistance pourrait apparaître en dernière analyse : l'exclusion symbolique (l'inscription dans le handicap) pourrait venir au secours de l'exclusion économique (l'inscription dans le chômage)! Le comble du paradoxe : c'est l'histoire d'un laotien, qui a fuit le régime communiste, et qui, après ses stages (non rémunérés) en entreprise, devant la difficulté de se faire embaucher en prospectant par ses propres moyens, s'étonne que la structure de formation ne lui trouve pas un emploi dans une entreprise certes privée, mais qui pourrait lui reverser une rémunération versée par l'État. Et vive l'Etat-providence ! 


\section{BIBLIOGRAPHIE}

Bui-Xuân G. (1987). - Influence d'une déficience sensorielle sur la représentation et la motricité. Revue STAPS, $\mathrm{n}^{\circ}$ spécial, 63-72.

Bui-Xuân G. (1987). - Les professeurs de judo auprès de personnes handicapées. Pratiques éducatives et positionnement social, Thèse de Doctorat STAPS, Université de Strasbourg II.

Bui-Xuân G. (1993). - Une modélisation du procès pédagogique. In Gilles Bui-Xuân et Jacques Gleyse (Eds). Enseigner l'éducation physique et sportive, Clermont-Ferrand, AFRAPS, 77-90.

Bui-Xuân G. (1994). - Gérer un curriculum conatif, $2^{\circ}$ Biennales de l'éducation et de la formation, Paris, serveur Minitel.

Bui-Xuân G. (1994). - Le cul est-il didactisable ? Tentative d'application d'un modèle pédagogique à l'éducation amoureuse. In Frédéric Baillette et Jean Marie Brohm. Traité critique d'éducation physique, Paris, Quel Corps ?, 189-199.

Demazière D. (1992). - Le chômage en crise ? La négociation des identités des chômeurs de longue durée, Lille, PUL.

Fougeyrollas P. (1993). - Les modèles explicatifs des conséquences des maladies et traumatismes : le processus de production des handicaps. Réseau International CIDIH, 6, 2, 14-28.

Goffman E. (1961). - Asylums, New-York, Doubleday. (Paris, Minuit pour l'édition française).

Malewska-Peyre H. (1990). - Le processus de dévalorisation de l'identité et les stratégies identitaires. In Carmel Camilleri et al. Stratégies identitaires, Paris, PUF.

Marcellini A. (1991). -Sport, Stigmate et intégration sociale : contribution à l'étude des stratégies de déstigmatisation, Thèse de Doctorat STAPS, Université de Montpellier I.

Mikulovic J. (1994). - Sentiment d'incompétence et stratégies identitaires. Intervention auprès d'un groupe de "travailleurs handicapés chômeurs de longue durée", Mémoire de DEA, UFR STAPS, Montpellier I.

Nirje B. (1980). - The normalization principle. In R.J. Flynn and K.E. Nitsch (Eds). Normalization, social integration, and community services, Baltimores, University Park Press, 31-49.

Sainsaulieu R. (1988). - L'identité au travail, Paris, Presses de la Fondation Nationale des Sciences Politiques.

Simard C., Binet M. et Dubé A. (1993). - Impact du sédentarisme et de l'activité physique sur les fonctions mentales : intégration avec la CIDIH. Journée d'étude Franco-Québécoise en A.P.A. Le Défi Sportif. (à paraître).

Simmel G. (1892). - Quelques réflexions sur la prostitution dans le présent et dans l'avenir. In Philosophie de l'amour, Paris, Rivages, 1988.

Tacussel P. (1993). - La figure sociale. Vers une sociologie des formes culturelles. Société, 40, 157-163.

Watzlawick P. et al. (1972). - Une logique de la communication, Paris, Seuil.

Wuhl S. (1992). - Les exclus face à l'emploi, Paris, Syros/Alternatives. 


\section{RÉSUMÉS}

$\mathrm{Au}$ cours de stages "d'orientation et d'adaptation à l'emploi" de travailleurs handicapés, chômeurs de longue durée, une intervention spécifique sur le CORPS semble avoir ouvert la voie à une redynamisation des sujets. Cette intervention a induit de nouveaux modèles, rejetant provisoirement les traditionnelles formations professionnelles qualifiantes, au profit de tout ce qui pousse le sujet à agir, et notamment la restauration d'un sentiment de compétence, une déculpabilisation, et un engagement autonome. Cette méthode a conduit ces personnes à un véritable reconditionnement à l'effort tant physique que sociétal. Il faut toutefois relativiser les succès de la réinsertion au regard des profils psychosociologiques qui président à des stratégies singulières, parfois subtiles, de préservation de soi.

During "employment's orientation and adaptation" training periods for disabled workers, long since unemployed, a specific intervention on the body seems to have opened the track to a subjects re-dynamisation process. This intervention has led to new patterns, temporarily throwing away the traditional qualifying professional's formations, in favour of everything which induces the subject to act, and especially the restoration of the self-efficacy, a guilt's obliteration, and a self-governing commitment. This approach has led people to get back into the habit of physical effort as well as societal effort. However, we need to bring a shade of meaning about the reinsertion success according to some psychosociological profiles which are linked with self-protection's specific strategies, sometimes subtle.

\section{INDEX}

Mots-clés : chômage

Keywords : conation, handicap, unemployement

\section{AUTEURS}

GILLES BUI-XUÂN

AFPA de Béziers

ANNE MARCELLINI

UFR STAPS, Université de Montpellier I

JACQUES MIKULOVIC

UFR STAPS, Université de Montpellier I 